Article

\title{
The Relationship between Dietary Habits and Frailty in Rural Japanese Community-Dwelling Older Adults: Cross-Sectional Observation Study Using a Brief Self-Administered Dietary History Questionnaire
}

\author{
Kayoko Tamaki ${ }^{1}$, Hiroshi Kusunoki ${ }^{1}$, Shotaro Tsuji ${ }^{2}$, Yosuke Wada ${ }^{3,4}$, Koutatsu Nagai ${ }^{5}$, \\ Masako Itoh ${ }^{5}$, Kyoko Sano ${ }^{5}$, Manabu Amano ${ }^{6}$, Hatsuo Maeda ${ }^{6}$, Yoko Hasegawa ${ }^{7}$, \\ Hiromitsu Kishimoto ${ }^{7}$, Sohji Shimomura ${ }^{3}$ and Ken Shinmura ${ }^{1,3, *}$ \\ 1 Division of General Medicine, Department of Internal Medicine, Hyogo College of Medicine, Nishinomiya, \\ Hyogo 663-8501, Japan; ka-tamaki@hyo-med.ac.jp (K.T.); kusunoki1019@yahoo.co.jp (H.K.) \\ 2 Department of Orthopedic Surgery, Hyogo College of Medicine Sasayama Medical Center, Sasayama, \\ Hyogo 669-2321, Japan; tj13041305sho@gmail.com \\ 3 Department of General Medicine and Community Health Science, Hyogo College of Medicine Sasayama \\ Medical Center, Sasayama, Hyogo 669-2321, Japan; yu-wada@hyo-med.ac.jp (Y.W.); \\ s-shimo@hyo-med.ac.jp (S.S.) \\ 4 Department of Rehabilitation, Hyogo College of Medicine Sasayama Medical Center, Sasayama, \\ Hyogo 669-2321, Japan \\ 5 School of Rehabilitation, Hyogo University of Health Sciences, Kobe, Hyogo 650-8530, Japan; \\ nagai-k@huhs.ac.jp (K.N.); masakoitoh@huhs.ac.jp (M.I.); kyokosano@huhs.ac.jp (K.S.) \\ 6 School of Pharmacy, Hyogo University of Health Sciences, Kobe, Hyogo 650-8530, Japan; \\ mbam@huhs.ac.jp (M.A.); hmaeda@huhs.ac.jp (H.M.) \\ 7 Department of Dentistry and Oral Surgery, Hyogo College of Medicine, Nishinomiya, Hyogo 663-8501, \\ Japan; cem17150@dent.niigata-u.ac.jp (Y.H.); kisihiro@hyo-med.ac.jp (H.K.) \\ * Correspondence: ke-shimmura@hyo-med.ac.jp; Tel.: +81-798-456-865; Fax: +81-798-456-977
}

Received: 31 October 2018; Accepted: 12 December 2018; Published: 14 December 2018

\begin{abstract}
To develop effective nutritional interventions for preventing frailty, the specific problems associated with the dietary habits of individuals based on sex differences must be identified. The purpose of this study was to evaluate the association between dietary habits and frailty in rural Japanese community-dwelling older adults. We recruited 800 participants, aged 65 and older, who underwent a comprehensive health examination between November 2015 and December 2017. Dietary habits were assessed by a brief self-administered dietary history questionnaire. Frailty was determined using either the Kihon Checklist (KCL) or the Japanese version of the Cardiovascular Health Study (J-CHS). The percentage of frail older adults was $8.4 \%$ according to KCL and $4.0 \%$ according to J-CHS. Various kinds of nutrient intakes, including three major nutrients, minerals, and vitamins in frail men, according to $\mathrm{KCL}$, were the lowest. By contrast, there were no differences in nutrient intake between the robust, prefrail, and frail female groups according to KCL. We found significant associations of the intakes of soluble dietary fiber, potassium, folate, and vitamin $\mathrm{C}$ with a frail status in men ( $p=0.035,0.023 .0 .012$, and 0.007 , respectively), and an association of the intake of vitamin $C$ with a frail status in women $(p=0.027)$ according to J-CHS. Attention should be paid to the diagnostic criteria of frailty and to sex differences, when nutritional interventions for the prevention of frailty are planned.
\end{abstract}

Keywords: dietary fiber; dietary habit; frailty; mineral; older adults; sex difference; vitamin 


\section{Introduction}

In aged societies, such as Japan, the establishment of management protocols for age-related diseases and long-term care, in connection with disability, are urgently required. In recent years, providing appropriate interventions for the elderly at the time of frailty onset has been considered important in the prevention of the progression of disability, as is the need for long-term care $[1,2]$. In the frailty cycle, the onset of muscle mass reduction (that is, presarcopenia) associated with aging and comorbidities, leads to muscle weakness and/or a decline in physical function (sarcopenia) as well as physical activity, which may result in the reduction of total energy expenditure [1-3]. The decrease in energy requirements also leads to the reduction of appetite and incidences of malnutrition and, eventually, reduction in the muscle mass, resulting in a vicious cycle of frailty [1-3]. Therefore, appropriate interventions should be implemented according to the phase of the frailty cycle.

To prevent the progression of frailty, interruption and slowing down of this vicious cycle are necessary. Lower nutritional status has been responsible for the progression of frailty [4,5]. Thus, appropriate interventions, in terms of diet and dietary behaviors, are necessary before the condition progresses to an irreversible status that requires long-term care. Previous studies have reported that increased protein intake and sufficient calorie intake are useful for the prevention and improvement of frail conditions [6-11]. In addition, high protein intake enhanced the protein anabolic response in frail individuals, resulting in an improvement in the negative nitrogen equilibrium [12]. To prevent age-associated reduction in lean mass, the recommended dietary allowance of protein of $0.8-1.0 \mathrm{~g} / \mathrm{kg} /$ day is insufficient - a protein allowance of $1.0-1.3 \mathrm{~g} / \mathrm{kg} /$ day is advisable [13]. Furthermore, a recent meta-analysis of observational studies demonstrated that low protein intake is closely associated with frailty in older adults $[9,14]$. In addition to proteins, micronutrients-such as vitamin $\mathrm{D}$, which is associated with bone metabolism, vitamins B6, C, and E, and folate that exerts antioxidant effects-were involved in frailty $[6,9,15]$. However, evidence regarding the association between dietary habits and frailty, which will help to establish appropriate nutritional interventions against frailty, is still lacking.

Frailty may be targeted to extend the healthy lifespan in community-dwelling older adults living at home. We hypothesized that nutritional interventions against frailty should be established, based on sex differences in dietary habits. Therefore, this cross-sectional observation study aimed to clarify the association between dietary habits and frailty diagnosed by two different methods in rural Japanese community-dwelling older adults using a self-administered dietary history questionnaire.

\section{Materials and Methods}

The cross-sectional study was designed as a Frail Elderly in the Sasayama-Tamba Area (FESTA) study. The study population, comprising of individuals $\geq 65$ years old, was recruited among community-dwelling elderly individuals in the Sasayama-Tamba area-a rural area in the Hyogo prefecture, Japan-between November 2015 and December 2017. This study was conducted in accordance with the Declaration of Helsinki, and the institutional review board of Hyogo College of Medicine approved the FESTA study, which complies with the current laws of Japan (approval No. Rinhi 0342). The survey commenced after an oral/written explanation of the study objectives, methods, and expected outcomes was provided to participants. All participants provided written informed consent. The data used in this study were anonymized and masked for analysis.

\subsection{Exclusion Criteria}

Of the 844 participants, we excluded individuals who displayed decreased cognitive function (Mini-Mental State Examination (MMSE) score less than 24) (males $n=7$, females $n=16$ ). In addition, individuals whose total energy intake, evaluated by a brief self-administered dietary history questionnaire (BDHQ), was less than $600 \mathrm{kcal} /$ day or more than 4,000 kcal/day were also excluded from further analyses (males $n=11$, females $n=10$ ). Therefore, 800 individuals were included in 
our analysis. The Kihon Checklist (KCL) analysis was performed in 800 participants (males $n=254$, females $n=546$ ). Analysis for the Cardiovascular Health Study (CHS) was performed in 796 older adults (males $n=252$, females $n=544$ ) after excluding individuals who failed to complete physical performance and movement tests (males $n=2$, females $n=2$ ). Analysis for the Asian working group for sarcopenia (AWGS) was performed in 788 older adults (males $n=250$, females $n=538$ ) after excluding individuals with electric devices implanted, including a pacemaker and a cardioverter-defibrillator (males $n=2$, females $n=6$ ).

\subsection{Measurement of Body Composition}

To evaluate muscle mass, participants were subjected to a bio-electrical impedance analysis (BIA) using InBody770 (Biospace Japan). Skeletal mass index (SMI) was defined as the muscle mass of extremities divided by the height squared. Testing was performed in the morning.

\subsection{Diagnosis of Frailty}

For the evaluation of frailty, we used two distinct criteria. A self-administered questionnaire survey was conducted using the KCL, which consists of 25 questions, and is used in screening participants who require care prevention in Japan [16]. KCL is divided into the following domains: instrumental activities of daily living (ADL), social ADL, exercise, falling, nutrition, oral function, cognitive function, and depression. Participants were asked to respond either "negative" (score: 1) or "positive" (score: 0), for a total score of 25. Frailty was determined using the methods introduced by Satake et al. [16]: frail, 8-25 points; pre-frail, 4-7 points, and robust, $0-3$ points.

We also characterized the frailty phenotype according to limitations in three or more of the following five conditions based on CHS: slow gait speed, weakness, exhaustion, low activity, and weight loss [17]. We scored the number of corresponding components as the frailty score, after modification (Japanese version of the CHS: J-CHS) [18]. Participants who did not have any of these components were considered as non-frail (robust), and those with one or two components were considered as prefrail. Slow gait speed was established based on a cutoff of $<1.0 \mathrm{~m} / \mathrm{s}$. Weakness was defined using maximum grip strength and was established according to a sex-specific cutoff ( $<26 \mathrm{~kg}$ for men and $<18 \mathrm{~kg}$ for women). Exhaustion was considered present if a participant responded "yes" to the following question included in the KCL: "In the last 2 weeks, have you felt tired without a reason?" We evaluated physical activity by asking the following questions about the time spent engaged in exercise: "Do you engage in low levels of physical exercise aimed at health?" If participants answered "no" to the questions, we classified them to the low activity category. Weight loss was assessed by a response of "yes" to the question, "Have you lost $2 \mathrm{~kg}$ or more in the past 6 months?"

\subsection{Dietary Survey}

Dietary habits during the preceding month were assessed using a brief-type self-administered diet history questionnaire (BDHQ)] [8,19]. The BDHQ for older people is a 10-page structured questionnaire used to inquire about the consumption frequencies of selected foods commonly consumed in Japan, general dietary behavior, and usual cooking methods. The daily intakes of 58 food items, energy, and selected nutrients were calculated using the BDHQ responses and an ad hoc computer algorithm based on the Standard Tables of Food Composition in Japan [20]. For all study participants, BDHQ calculations were made using fixed, sex-specific portion sizes. The methods used to calculate the dietary intakes and the validity of the BDHQ have been detailed elsewhere [8,19]. Energy intake distribution was determined based on the calculated nutrient intake by using the residual method. All participants answered a BDHQ by themselves, and a trained managerial dietician or investigator helped them complete all questionnaires. 


\subsection{Statistical Analysis}

The results were expressed as mean \pm standard deviation for continuous variables. Categorical variables were expressed as percentages. To compare participants' characteristics, an unpaired $t$ test and a $\chi^{2}$ test were used for comparisons of continuous and categorical variables, respectively. For intergroup comparisons, data were analyzed using a $\chi^{2}$ test and one-way analysis of variance (ANOVA), followed by the Tukey-Kramer test. To determine significant differences in 4 dietary nutrients (soluble dietary fiber, potassium, folate, and vitamin C) between robust, prefrail, and frail individuals after excluding the possible confounding effects of baseline differences, an analysis of covariance (ANCOVA), adjusting for age, BMI, smoking habit, and alcohol consumption, was conducted. For each statistically significant effect of a dietary nutrient, the Bonferroni post hoc test was executed. $p$-values $<0.05$ were considered statistically significant. JMP 13.1 software was used for data analysis.

\section{Results}

\subsection{Basic Characteristics and the Prevalence of Frailty}

The average age of the 800 participants was $72.6 \pm 5.8$ years. Of these, 254 were male $(31.8 \%)$ and 546 were female $(68.3 \%) ; 529(66.1 \%)$ were young-old (65-74 years old) and $271(33.9 \%)$ were old-old ( $\geq 75$ years old). As shown in Table 1, there was no significant difference between men and women in terms of age, blood pressure, MMSE score, and exercise habits. However, height, body weight (BW), and body mass index (BMI), were higher in men than in women. There was a higher number of current smokers and alcohol drinkers among men. We also found differences between sexes in the proportions of comorbidities, such as hypertension, diabetes mellitus (DM), dyslipidemia, cardiovascular disease (CVD), osteoporosis, and a history of cancer.

Table 1. Basic characteristics and the diagnosis of frailty in the 800 participants.

\begin{tabular}{|c|c|c|c|c|}
\hline & Total $(n=800)$ & Male $(n=254)$ & Female $(n=546)$ & $p$-Value \\
\hline Age (years) & $72.6 \pm 5.8$ & $73.1 \pm 6.2$ & $72.4 \pm 5.6$ & n.s. \\
\hline Height (cm) & $155.5 \pm 8.1$ & $164.1 \pm 5.8$ & $151.5 \pm 5.6$ & $<0.001$ \\
\hline BW $(\mathrm{kg})$ & $54.9 \pm 9.4$ & $62.7 \pm 9.0$ & $51.3 \pm 7.1$ & $<0.001$ \\
\hline $\mathrm{BMI}\left(\mathrm{kg} / \mathrm{m}^{2}\right)$ & $22.6 \pm 2.9$ & $23.2 \pm 2.9$ & $22.3 \pm 2.9$ & $<0.001$ \\
\hline $\mathrm{SBP}(\mathrm{mmHg})$ & $138.9 \pm 17.6$ & $137.5 \pm 18.2$ & $139.6 \pm 17.4$ & n.s. \\
\hline DBP $(\mathrm{mmHg})$ & $80.5 \pm 10.7$ & $80.3 \pm 11.4$ & $80.6 \pm 10.3$ & n.s. \\
\hline MMSE score & $28.3 \pm 1.8$ & $28.2 \pm 1.8$ & $28.4 \pm 1.8$ & n.s. \\
\hline \multicolumn{5}{|l|}{ Smoking habit } \\
\hline Never & $579(72.4)$ & $71(28.0)$ & $508(93.0)$ & \\
\hline Past & $181(22.6)$ & $155(61.0)$ & $26(4.8)$ & \\
\hline Current & $40(5.0)$ & $28(11.0)$ & $12(2.2)$ & $<0.001$ \\
\hline Alcohol drinking & $349(43.6)$ & $192(75.6)$ & $157(28.8)$ & $<0.001$ \\
\hline Exercise habit & $508(63.5)$ & $154(60.6)$ & $354(64.8)$ & n.s. \\
\hline \multicolumn{5}{|l|}{ Comorbidities } \\
\hline Hypertension & $362(45.3)$ & $128(50.4)$ & $234(42.9)$ & 0.046 \\
\hline DM & $98(12.3)$ & $50(19.7)$ & $48(8.8)$ & $<0.001$ \\
\hline Dyslipidemia & $192(24.0)$ & $44(17.3)$ & $148(27.1)$ & 0.003 \\
\hline Chronic liver disease & $34(4.3)$ & $16(6.3)$ & $18(3.3)$ & n.s. \\
\hline CKD & $27(3.4)$ & $11(4.3)$ & $16(2.9)$ & n.s. \\
\hline CVD & $70(8.8)$ & $34(13.4)$ & $36(6.6)$ & 0.002 \\
\hline Gastrointestinal disease & $48(6.0)$ & $18(7.1)$ & $30(5.5)$ & n.s. \\
\hline Asthma and COPD & $24(3.0)$ & $7(2.8)$ & $17(3.1)$ & n.s. \\
\hline Thyroid disease & $35(4.4)$ & $8(3.1)$ & $27(4.9)$ & n.s. \\
\hline Osteoporosis & $97(12.1)$ & $3(1.2)$ & $94(17.2)$ & $<0.001$ \\
\hline RA and collagen disease & $17(2.1)$ & $4(1.6)$ & $13(2.4)$ & n.s. \\
\hline History of cancer & $58(7.3)$ & $30(11.8)$ & $28(4.2)$ & $<0.001$ \\
\hline Frailty diagnosis by the KCL criteria & $(n=800)$ & $(n=254)$ & $(n=546)$ & \\
\hline Robust & 476 (59.5) & $163(64.3)$ & 313 (57.3) & \\
\hline
\end{tabular}


Table 1. Cont.

\begin{tabular}{ccccc}
\hline & Total $(\boldsymbol{n}=\mathbf{8 0 0})$ & Male $(\boldsymbol{n = 2 5 4 )}$ & Female $(\boldsymbol{n}=\mathbf{5 4 6})$ & $\boldsymbol{p}$-Value \\
\hline Prefrail & $257(32.1)$ & $72(28.2)$ & $185(33.9)$ & \\
Frail & $67(8.4)$ & $19(7.5)$ & $48(8.8)$ & n.s. \\
Frailty diagnosis by the J-CHS criteria & $(n=796)$ & $(n=252)$ & $(n=544)$ & \\
Robust & $331(41.6)$ & $104(41.3)$ & $227(41.7)$ & \\
Prefrail & $436(54.8)$ & $139(55.2)$ & $294(54.0)$ & \\
Frail & $32(4.0)$ & $9(3.6)$ & $23(4.2)$ & n.s. \\
Sarcopenia diagnosis by the AWGS & $(n=788)$ & $(n=250)$ & $(n=538)$ & \\
criteria & $542(68.8)$ & $182(72.8)$ & $360(66.9)$ & \\
Robust & $217(27.5)$ & $65(26.0)$ & $152(28.3)$ & \\
Presarcopenia & $29(3.7)$ & $3(1.2)$ & $26(4.8)$ & 0.026 \\
Sarcopenia &
\end{tabular}

The data in the table represent the prevalence of a condition by the total number of individuals (mean \pm SD or number), with \% of population in parenthesis next to it. This applies to all variables unless specific units are provided. BW: body weight, BMI: body mass index, SBP: systolic blood pressure, DBP: diastolic blood pressure, MMSE: Mini-Mental State Examination, DM: diabetes mellitus, CKD: chronic kidney disease, CVD: cardiovascular disease, COPD: cardiopulmonary disease, RA: rheumatoid arthritis, KCL: Kihon Checklist, J-CHS: Japanese version of the Cardiovascular Health Study, AWGS: Asian Working Group for Sarcopenia.

As for frailty severity, in this study, $59.5 \%$ of the participants were healthy (robust), $32.1 \%$ prefrail, and $8.4 \%$ frail, according to the KCL criteria, and $41.6 \%$ were robust, $54.8 \%$ prefrail, and $4.0 \%$ frail, according to the J-CHS criteria. The concordance rate for the two diagnoses was $57.0 \%$. In terms of the diagnosis of sarcopenia, $68.8 \%$ of the participants were robust, $27.5 \%$ had presarcopenia, and $3.7 \%$ had sarcopenia. Although there was no difference in the prevalence of frailty between men and women, the prevalence of sarcopenia was significantly higher in women than in men (Table 1).

There were significant differences between men and women in terms of body size, as well as smoking and alcohol consumption status. Therefore, nutritional analyses were performed separately in men and women. In addition, we compared basic characteristics, nutrient intake, and dietary habits between robust, prefrail, and frail individuals, because the prevalence of sarcopenia in men was too low to obtain meaningful analysis results.

\subsection{Comparison of the Basic Characteristics, Nutrient Intake, and Dietary Habits between Robust, Prefrail, and Frail Individuals, Diagnosed by the KCL Criteria}

As shown in Table 2, the prefrail male group, diagnosed by the KCL criteria, was older, compared with the robust male group. However, there were no differences in other basic characteristics between the three groups.

Table 2. Basic characteristics and dietary habits among frail, prefrail, and robust men, as diagnosed by the KCL criteria.

\begin{tabular}{|c|c|c|c|c|}
\hline & Robust ( $n=163$ ) & Prefrail $(n=72)$ & Frail $(n=19)$ & $p$-Value \\
\hline Height (cm) & $164.3 \pm 5.8$ & $163.6 \pm 6.4$ & $163.7 \pm 4.0$ & n.s. \\
\hline $\mathrm{BMI}\left(\mathrm{kg} / \mathrm{m}^{2}\right)$ & $23.4 \pm 2.8$ & $23.1 \pm 3.2$ & $22.4 \pm 3.1$ & n.s. \\
\hline SBP (mmHg) & $137.5 \pm 19.0$ & $136.9 \pm 15.9$ & $138.7 \pm 19.8$ & n.s. \\
\hline $\mathrm{DBP}(\mathrm{mmHg})$ & $81.4 \pm 11.6$ & $77.7 \pm 10.6$ & $80.9 \pm 11.3$ & n.s. \\
\hline Never & $48(29.4)$ & $18(25.0)$ & $5(26.3)$ & \\
\hline Past & $98(60.1)$ & $49(68.1)$ & $8(42.1)$ & \\
\hline Current & $17(10.4)$ & $5(6.9)$ & $6(31.6)$ & 0.033 \\
\hline Alcohol drinking & $126(77.3)$ & $51(70.8)$ & $15(78.9)$ & n.s. \\
\hline Exercise habit & $106(65.0)$ & $39(54.2)$ & $9(47.4)$ & n.s. \\
\hline
\end{tabular}


Table 2. Cont.

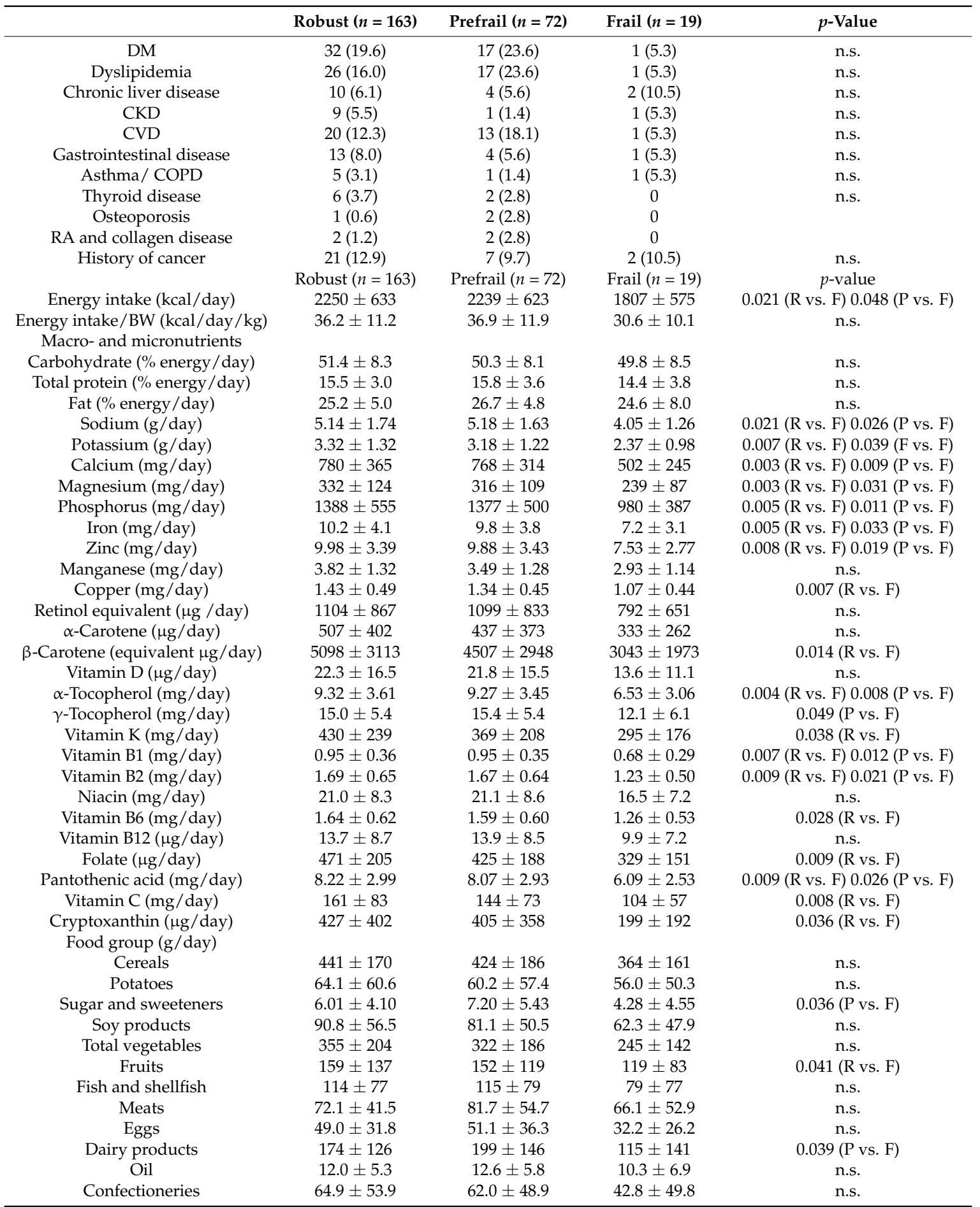

Mean \pm SD or number (\%), n.s.: not significant, R: Robust, P: Prefrail, F: Frail.

The carbohydrate, total protein (both animal and vegetable) and fat (both animal and vegetable) intake in the frail male group was the lowest among the three groups (Figure 1A-E). In addition, the intake of dietary fiber, both soluble and insoluble, was the lowest in the frail male group (Figure 1F). Among various nutrients, the intakes of sodium, potassium, calcium, magnesium, phosphorus, iron, zinc, $\alpha$-tocopherol, vitamins B1 and B2, and pantothenic acid were the lowest in the frail male group (Table 2). The intake of copper, $\beta$-carotene (equivalent), $\gamma$-tocopherol, vitamins B6, $C$, and $K$, folate, and cryptoxanthin were lower in the frail male group than in the robust male group. In terms of food 
groups, the intake of sugar and sweeteners, and dairy products was significantly lower in the frail male group than in the prefrail male group.

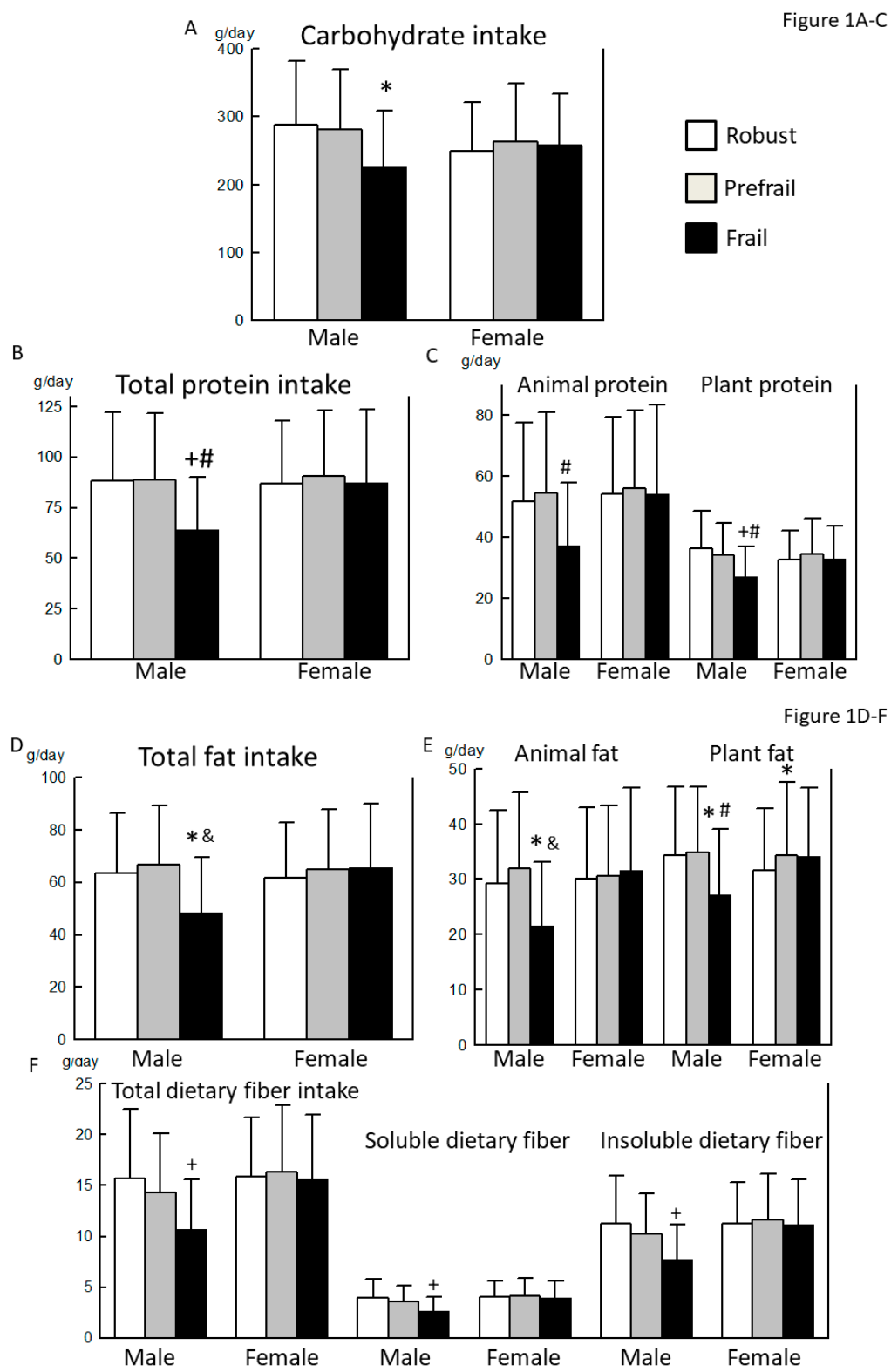

Figure 1. Comparison of macronutrient intake between robust, prefrail, and frail individuals, diagnosed by the Kihon checklist. (A): Carbohydrate intake, (B): Total protein intake, (C): Animal and plant protein intake, (D): Total fat intake, (E): Animal and plant fat intake, and (F): Total, soluble, and insoluble dietary fiber intake. $*: p<0.05$ vs. Robust, $+: p<0.01$ vs. Robust, \#: $p<0.05$ vs. Prefrail, \&: $p<0.01$ vs. Prefrail.

As shown in Table 3, age was the highest and height was the lowest in the frail female group. Age was significantly higher in the prefrail female group than in the robust female group. In addition, the frail female group showed lower alcohol consumption and lower exercise status. The proportion of individuals with chronic kidney disease (CKD), gastrointestinal disease, asthma/chronic obstructive pulmonary disease (COPD), osteoporosis, and a history of cancer was significantly higher in the frail female group, compared with the robust female group. 
Table 3. Basic characteristics and dietary habits among frail, prefrail, and robust women, as diagnosed by the KCL criteria.

\begin{tabular}{|c|c|c|c|c|}
\hline & Robust $(n=313)$ & Prefrail $(n=185)$ & Frail $(n=48)$ & $p$-Value \\
\hline Age (years) & $71.7 \pm 5.5$ & $72.9 \pm 5.6$ & $75.4 \pm 5.7$ & $\begin{array}{c}<0.001 \text { (R vs. F) } 0.042 \text { (R vs. P) } \\
0.018 \text { (P vs. F) }\end{array}$ \\
\hline Height $(\mathrm{cm})$ & $152.1 \pm 5.3$ & $151.2 \pm 5.7$ & $148.5 \pm 6.2$ & $<0.001$ (R vs. F) 0.009 (P vs. F) \\
\hline BW (kg) & $51.8 \pm 7.0$ & $50.6 \pm 7.1$ & $50.4 \pm 7.6$ & n.s. \\
\hline BMI $\left(\mathrm{kg} / \mathrm{m}^{2}\right)$ & $22.4 \pm 2.7$ & $22.2 \pm 3.0$ & $22.8 \pm 3.5$ & n.s. \\
\hline SBP (mmHg) & $139.5 \pm 16.6$ & $139.1 \pm 18.5$ & $142.3 \pm 17.9$ & n.s. \\
\hline $\mathrm{DBP}(\mathrm{mmHg})$ & $80.8 \pm 10.1$ & $80.4 \pm 10.9$ & $80.6 \pm 9.6$ & n.s. \\
\hline MMSE score & $28.5 \pm 1.8$ & $28.4 \pm 1.8$ & $28.0 \pm 1.8$ & n.s. \\
\hline Smoking Habit Never & $293(93.6)$ & $170(91.9)$ & $45(93.8)$ & \\
\hline Past & $13(4.2)$ & $11(5.9)$ & $2(4.2)$ & \\
\hline Current & $7(2.2)$ & $4(2.2)$ & $1(2.1)$ & n.s. \\
\hline Alcohol drinking & $99(31.6)$ & $52(28.1)$ & $6(12.5)$ & 0.024 \\
\hline Exercise habit & $215(68.7)$ & $119(64.3)$ & $20(41.7)$ & 0.001 \\
\hline \multicolumn{5}{|l|}{ Comorbidities } \\
\hline Hypertension & $125(39.9)$ & $84(45.4)$ & $25(52.1)$ & n.s. \\
\hline DM & $24(7.7)$ & $17(9.2)$ & $7(14.6)$ & n.s. \\
\hline Dyslipidemia & $88(28.1)$ & $49(26.5)$ & $11(22.9)$ & n.s. \\
\hline Chronic liver disease & $11(3.5)$ & $4(2.2)$ & $3(6.3)$ & n.s. \\
\hline CKD & $6(1.9)$ & $5(2.7)$ & $5(10.4)$ & 0.005 \\
\hline CVD & $18(5.8)$ & $16(8.6)$ & $4(8.3)$ & n.s. \\
\hline Gastrointestinal disease & $13(4.2)$ & $9(4.9)$ & $8(16.7)$ & 0.002 \\
\hline Asthma/ COPD & $4(1.3)$ & $10(5.4)$ & $3(6.3)$ & 0.016 \\
\hline Thyroid disease & $13(4.2)$ & $13(7.0)$ & $1(2.1)$ & n.s. \\
\hline Osteoporosis & $42(13.4)$ & $40(21.6)$ & $12(25.0)$ & 0.021 \\
\hline RA and collagen disease & $9(2.9)$ & $4(2.2)$ & 0 & \\
\hline \multirow[t]{2}{*}{ History of cancer } & $12(3.8)$ & $10(5.4)$ & $6(12.5)$ & 0.039 \\
\hline & Robust $(n=313)$ & Prefrail $(n=185)$ & Frail $(n=48)$ & $p$-value \\
\hline Energy intake (kcal/day) & $1928 \pm 537$ & $2032 \pm 594$ & $1994 \pm 624$ & 0.031 (R vs. P) \\
\hline $\begin{array}{c}\text { Energy intake/BW (kcal/day/kg) } \\
\text { Macro- and micronutrients }\end{array}$ & $38.0 \pm 12.3$ & $40.9 \pm 13.4$ & $40.4 \pm 13.6$ & n.s. \\
\hline Carbohydrate (\% energy/day) & $51.9 \pm 7.0$ & $51.9 \pm 7.7$ & $52.2 \pm 6.7$ & n.s. \\
\hline Total protein (\% energy/day) & $18.0 \pm 3.3$ & $17.8 \pm 3.5$ & $17.2 \pm 3.1$ & n.s. \\
\hline Fat (\% energy/day) & $28.7 \pm 4.8$ & $28.6 \pm 5.2$ & $29.5 \pm 4.8$ & n.s. \\
\hline Sodium (g/day) & $4.66 \pm 1.53$ & $4.92 \pm 1.56$ & $4.71 \pm 1.77$ & n.s. \\
\hline Potassium (g/day) & $3.41 \pm 1.14$ & $3.52 \pm 1.29$ & $3.33 \pm 1.27$ & n.s. \\
\hline Calcium (mg/day) & $790 \pm 291$ & $827 \pm 336$ & $737 \pm 272$ & n.s. \\
\hline Magnesium (mg/day) & $321 \pm 105$ & $334 \pm 115$ & $311 \pm 114$ & n.s. \\
\hline Phosphorus (mg/day) & $1364 \pm 483$ & $1422 \pm 519$ & $1340 \pm 542$ & n.s. \\
\hline Iron (mg/day) & $10.2 \pm 3.5$ & $10.5 \pm 3.6$ & $10.1 \pm 3.8$ & n.s. \\
\hline Zinc (mg/day) & $9.56 \pm 3.07$ & $9.90 \pm 3.08$ & $9.58 \pm 3.55$ & n.s. \\
\hline Manganese (mg/day) & $3.86 \pm 1.11$ & $3.92 \pm 1.22$ & $3.70 \pm 1.20$ & n.s. \\
\hline Copper (mg/day) & $1.34 \pm 0.40$ & $1.39 \pm 0.45$ & $1.35 \pm 0.47$ & n.s. \\
\hline Retinol equivalent ( $\mu \mathrm{g} /$ day) & $1019 \pm 688$ & $1018 \pm 586$ & $934 \pm 478$ & n.s. \\
\hline$\alpha$-Carotene $(\mu \mathrm{g} / \mathrm{day})$ & $649 \pm 423$ & $689 \pm 490$ & $592 \pm 399$ & n.s. \\
\hline$\beta$-Carotene equivalent ( $\mu \mathrm{g} /$ day) & $5820 \pm 3188$ & $6076 \pm 3433$ & $5558 \pm 3201$ & n.s. \\
\hline Vitamin D ( $\mu \mathrm{g} /$ day) & $24.1 \pm 15.9$ & $25.7 \pm 16.0$ & $22.2 \pm 16.1$ & n.s. \\
\hline$\alpha$-Tocopherol (mg/day) & $9.44 \pm 3.37$ & $9.93 \pm 3.69$ & $9.86 \pm 4.16$ & n.s. \\
\hline$\gamma$-Tocopherol (mg/day) & $13.6 \pm 4.8$ & $14.7 \pm 5.7$ & $14.7 \pm 5.3$ & n.s. \\
\hline Vitamin K (mg/day) & $413 \pm 202$ & $423 \pm 216$ & $399 \pm 232$ & n.s. \\
\hline Vitamin B1 (mg/day) & $0.98 \pm 0.33$ & $1.01 \pm 0.35$ & $0.97 \pm 0.38$ & n.s. \\
\hline Vitamin B2 (mg/day) & $1.70 \pm 0.54$ & $1.73 \pm 0.59$ & $1.66 \pm 0.57$ & n.s. \\
\hline Niacin (mg/day) & $21.3 \pm 8.5$ & $21.9 \pm 8.2$ & $20.9 \pm 9.8$ & n.s. \\
\hline Vitamin B6 (mg/day) & $1.63 \pm 0.59$ & $1.67 \pm 0.61$ & $1.62 \pm 0.68$ & n.s. \\
\hline Vitamin B12 ( $\mu \mathrm{g} /$ day $)$ & $13.7 \pm 8.1$ & $14.4 \pm 8.2$ & $13.3 \pm 9.1$ & n.s. \\
\hline Folate ( $\mu \mathrm{g} /$ day) & $479 \pm 178$ & $484 \pm 188$ & $454 \pm 185$ & n.s. \\
\hline Pantothenic acid (mg/day) & $8.00 \pm 2.59$ & $8.20 \pm 2.83$ & $7.99 \pm 2.97$ & n.s. \\
\hline Vitamin C (mg/day) & $182 \pm 75$ & $183 \pm 84$ & $175 \pm 78$ & n.s. \\
\hline Cryptoxanthin ( $\mu \mathrm{g} /$ day $)$ & $489 \pm 352$ & $519 \pm 437$ & $454 \pm 331$ & n.s. \\
\hline \multicolumn{5}{|l|}{ Food group (g/day) } \\
\hline Cereals & $336 \pm 142$ & $356 \pm 147$ & $348 \pm 139$ & n.s. \\
\hline Potatoes & $68.9 \pm 53.7$ & $73.0 \pm 62.2$ & $74.3 \pm 62.9$ & n.s. \\
\hline Sugar and sweeteners & $5.90 \pm 4.15$ & $6.39 \pm 5.03$ & $6.48 \pm 4.55$ & n.s. \\
\hline Soy products & $88.0 \pm 45.8$ & $91.6 \pm 54.1$ & $84.0 \pm 46.3$ & n.s. \\
\hline Total vegetables & $377 \pm 186$ & $392 \pm 201$ & $365 \pm 184$ & n.s. \\
\hline Fruits & $180 \pm 114$ & $180 \pm 135$ & $178 \pm 116$ & n.s. \\
\hline Fish and shellfish & $120 \pm 76$ & $128 \pm 79$ & $120 \pm 100$ & n.s. \\
\hline Meats & $79.9 \pm 51.5$ & $77.3 \pm 39.2$ & $81.6 \pm 60.6$ & n.s. \\
\hline Eggs & $43.9 \pm 26.3$ & $44.8 \pm 31.0$ & $54.0 \pm 35.6$ & n.s. \\
\hline Dairy products & $174 \pm 97$ & $179 \pm 119$ & $159 \pm 88$ & n.s. \\
\hline Oil & $10.0 \pm 5.0$ & $11.0 \pm 5.4$ & $12.0 \pm 5.4$ & 0.036 (R vs. F) \\
\hline Confectioneries & $67.7 \pm 50.2$ & $73.3 \pm 63.4$ & $76.6 \pm 49.0$ & n.s. \\
\hline
\end{tabular}


The plant fat intake was significantly higher in the prefrail female group than in the robust female group (Figure 1E). There was no significant difference in the intake of other nutrients between the three groups (Figure 1A-F and Table 3). In terms of food groups, there was no significant difference among the 3 groups, except that oil intake was higher in the frail female group than in the robust female group.

3.3. Comparison of the Basic Characteristics, Nutrient Intake, and Dietary Habits between Robust, Prefrail, and Frail Individuals, Diagnosed by the J-CHS Criteria

As shown in Table 4, no statistically significant differences in the basic characteristics were observed between the robust, prefrail, and frail male groups, diagnosed by the J-CHS criteria, except for the exercise status.

Table 4. Basic characteristics and dietary habits among frail, prefrail, and robust men, as diagnosed by the J-CHS criteria.

\begin{tabular}{|c|c|c|c|c|}
\hline & Robust $(n=104)$ & Prefrail $(n=139)$ & Frail $(n=9)$ & $p$-Value \\
\hline Age (years) & $73.4 \pm 5.0$ & $72.8 \pm 7.0$ & $74.9 \pm 7.0$ & n.s. \\
\hline Height (cm) & $164.4 \pm 6.0$ & $163.8 \pm 5.8$ & $165.6 \pm 5.4$ & n.s. \\
\hline BW $(\mathrm{kg})$ & $62.6 \pm 8.7$ & $62.8 \pm 9.1$ & $61.4 \pm 11.0$ & n.s. \\
\hline BMI $\left(\mathrm{kg} / \mathrm{m}^{2}\right)$ & $23.2 \pm 2.9$ & $23.4 \pm 2.9$ & $22.4 \pm 4.2$ & n.s. \\
\hline $\mathrm{SBP}(\mathrm{mmHg})$ & $136.3 \pm 16.5$ & $137.8 \pm 19.6$ & $142.6 \pm 14.0$ & n.s. \\
\hline DBP (mmHg) & $78.7 \pm 10.9$ & $81.3 \pm 11.8$ & $81.9 \pm 10.0$ & n.s. \\
\hline MMSE score & $28.2 \pm 1.9$ & $28.1 \pm 1.9$ & $28.3 \pm 1.8$ & n.s. \\
\hline \multicolumn{5}{|l|}{ Smoking Habit } \\
\hline Never & $30(28.8)$ & $39(28.3)$ & $2(22.2)$ & \\
\hline Past & $65(62.5)$ & $83(59.7)$ & $5(55.6)$ & \\
\hline Current & $9(8.7)$ & $17(12.2)$ & $2(22.2)$ & n.s. \\
\hline Alcohol drinking & $76(73.1)$ & $108(77.7)$ & $7(77.8)$ & n.s. \\
\hline Exercise habit & $104(100.0)$ & $50(75.2)$ & 0 & \\
\hline \multicolumn{5}{|l|}{ Comorbidities } \\
\hline Hypertension & $45(43.3)$ & $77(55.4)$ & $5(55.6)$ & n.s. \\
\hline $\mathrm{DM}$ & $26(25.0)$ & $20(14.4)$ & $3(33.3)$ & n.s. \\
\hline Dyslipidemia & $19(18.3)$ & $24(17.3)$ & 0 & \\
\hline Chronic liver disease & $9(8.7)$ & $7(5.0)$ & 0 & \\
\hline CKD & $6(5.8)$ & $4(2.9)$ & $1(11.1)$ & n.s. \\
\hline CVD & $17(16.3)$ & $17(12.2)$ & 0 & \\
\hline Gastrointestinal disease & $9(8.7)$ & $9(6.5)$ & 0 & \\
\hline Asthma/ COPD & $4(3.8)$ & $2(1.4)$ & $1(11.1)$ & n.s. \\
\hline Thyroid disease & $4(3.8)$ & $4(2.9)$ & 0 & \\
\hline Osteoporosis & $1(1.0)$ & $1(0.7)$ & $1(11.1)$ & 0.02 \\
\hline RA and collagen disease & $1(1.0)$ & $3(2.2)$ & 0 & \\
\hline \multirow[t]{2}{*}{ History of cancer } & $14(13.5)$ & $14(10.1)$ & $2(22.2)$ & n.s. \\
\hline & Robust $(n=104)$ & Prefrail $(n=139)$ & Frail $(n=9)$ & $p$-value \\
\hline Energy intake (kcal/day) & $2305 \pm 605$ & $2170 \pm 644$ & $1809 \pm 567$ & n.s. \\
\hline Energy intake/BW (kcal/day/kg) & $37.4 \pm 11.0$ & $35.2 \pm 11.5$ & $30.6 \pm 11.6$ & n.s. \\
\hline Macro- and micronutrients & & & & \\
\hline Carbohydrate (\% energy/day) & $50.7 \pm 7.9$ & $51.6 \pm 8.4$ & $48.4 \pm 9.2$ & n.s. \\
\hline Total protein (\% energy/day) & $15.8 \pm 3.0$ & $15.2 \pm 3.3$ & $16.6 \pm 4.4$ & n.s. \\
\hline Fat (\% energy/day) & $25.9 \pm 4.6$ & $25.1 \pm 5.6$ & $28.0 \pm 6.7$ & n.s. \\
\hline Sodium (g/day) & $5.26 \pm 1.63$ & $4.93 \pm 1.75$ & $4.71 \pm 1.44$ & n.s. \\
\hline Potassium (g/day) & $3.47 \pm 1.31$ & $3.04 \pm 1.25$ & $2.64 \pm 1.29$ & 0.029 (R vs. P) \\
\hline Calcium (mg/day) & $814 \pm 354$ & $723 \pm 344$ & $560 \pm 325$ & n.s. \\
\hline Magnesium (mg/day) & $342 \pm 123$ & $308 \pm 116$ & $265 \pm 95$ & n.s. \\
\hline Phosphorus (mg/day) & $1440 \pm 535$ & $1300 \pm 532$ & $1156 \pm 472$ & n.s. \\
\hline Iron (mg/day) & $10.68 \pm 4.03$ & $9.32 \pm 3.44$ & $8.15 \pm 2.92$ & 0.023 (R vs. P) \\
\hline Zinc (mg/day) & $10.29 \pm 3.36$ & $9.46 \pm 3.44$ & $8.53 \pm 2.68$ & n.s. \\
\hline Manganese (mg/day) & $3.83 \pm 1.29$ & $3.60 \pm 1.34$ & $2.78 \pm 0.90$ & n.s. \\
\hline Copper (mg/day) & $1.46 \pm 0.50$ & $1.33 \pm 0.47$ & $1.15 \pm 0.41$ & n.s. \\
\hline Retinol equivalent ( $\mu \mathrm{g} /$ day) & $1210 \pm 1028$ & $984 \pm 665$ & $1094 \pm 946$ & n.s. \\
\hline$\alpha$-Carotene ( $\mu \mathrm{g} /$ day) & $530 \pm 400$ & $440 \pm 371$ & $420 \pm 479$ & n.s. \\
\hline$\beta$-Carotene equivalent ( $\mu \mathrm{g} /$ day) & $5405 \pm 3113$ & $4401 \pm 2930$ & $3742 \pm 3093$ & 0.029 (R vs. P) \\
\hline Vitamin D ( $\mu \mathrm{g} /$ day $)$ & $22.9 \pm 16.4$ & $20.3 \pm 15.3$ & $19.0 \pm 14.6$ & n.s. \\
\hline$\alpha$-Tocopherol (mg/day) & $9.69 \pm 3.41$ & $8.69 \pm 3.69$ & $7.80 \pm 2.80$ & n.s. \\
\hline$\gamma$-Tocopherol (mg/day) & $15.2 \pm 5.2$ & $14.7 \pm 5.7$ & $13.9 \pm 4.6$ & n.s. \\
\hline Vitamin K (mg/day) & $443 \pm 238$ & $380 \pm 224$ & $313 \pm 140$ & n.s. \\
\hline Vitamin B1 (mg/day) & $1.00 \pm 0.35$ & $0.88 \pm 0.36$ & $0.81 \pm 0.38$ & 0.024 (R vs. P) \\
\hline
\end{tabular}


Table 4. Cont.

\begin{tabular}{|c|c|c|c|c|}
\hline & Robust $(n=104)$ & Prefrail $(n=139)$ & Frail $(n=9)$ & $p$-Value \\
\hline Vitamin B2 (mg/day) & $1.78 \pm 0.65$ & $1.57 \pm 0.63$ & $1.33 \pm 0.56$ & 0.032 (R vs. P) \\
\hline Niacin (mg/day) & $22.0 \pm 8.5$ & $19.8 \pm 8.3$ & $17.7 \pm 6.5$ & n.s. \\
\hline Vitamin B6 (mg/day) & $1.72 \pm 0.61$ & $1.52 \pm 0.60$ & $1.27 \pm 0.60$ & 0.026 (R vs. $P$ ) \\
\hline Vitamin B12 ( $\mu \mathrm{g} /$ day $)$ & $14.4 \pm 8.8$ & $12.7 \pm 8.3$ & $11.5 \pm 6.5$ & n.s. \\
\hline Folate ( $\mu \mathrm{g} /$ day) & $493 \pm 208$ & $419 \pm 190$ & $365 \pm 175$ & 0.011 (R vs. P) \\
\hline Pantothenic acid (mg/day) & $8.60 \pm 3.01$ & $7.67 \pm 2.93$ & $6.80 \pm 2.61$ & 0.043 (R vs. P) \\
\hline Vitamin C (mg/day) & $171 \pm 82$ & $140 \pm 76$ & $118 \pm 90$ & 0.008 (R vs. P) \\
\hline Cryptoxanthin ( $\mu \mathrm{g} /$ day) & $484 \pm 429$ & $358 \pm 325$ & $358 \pm 455$ & n.s. \\
\hline \multicolumn{5}{|l|}{ Food group (g/day) } \\
\hline Cereals & $427 \pm 166$ & $438 \pm 181$ & $381 \pm 178$ & n.s. \\
\hline Potatoes & $70.2 \pm 61.5$ & $56.7 \pm 54.9$ & $65.0 \pm 82.7$ & n.s. \\
\hline Sugar and sweeteners & $6.47 \pm 4.20$ & $5.93 \pm 4.62$ & $6.95 \pm 7.11$ & n.s. \\
\hline Soy products & $93.1 \pm 59.9$ & $81.4 \pm 48.9$ & $61.0 \pm 34.0$ & n.s. \\
\hline Total vegetables & $369 \pm 194$ & $317 \pm 197$ & $281 \pm 206$ & n.s. \\
\hline Fruits & $172 \pm 150$ & $137 \pm 109$ & $107 \pm 104$ & n.s. \\
\hline Fish and shellfish & $118 \pm 78$ & $106 \pm 74$ & $96 \pm 60$ & n.s. \\
\hline Meats & $80.1 \pm 48.1$ & $70.1 \pm 46.3$ & $76.7 \pm 30.5$ & n.s. \\
\hline Eggs & $51.2 \pm 29.7$ & $45.9 \pm 33.8$ & $45.0 \pm 35.1$ & n.s. \\
\hline Dairy products & $188 \pm 122$ & $174 \pm 144$ & $122 \pm 101$ & n.s. \\
\hline Oil & $12.1 \pm 5.4$ & $12.0 \pm 5.7$ & $11.7 \pm 5.3$ & n.s. \\
\hline Confectioneries & $70.7 \pm 56.1$ & $59.0 \pm 49.9$ & $30.7 \pm 20.3$ & 0.038 (R vs. F) \\
\hline
\end{tabular}

Mean \pm SD or number (\%), n.s.: not significant, R: Robust, P: Prefrail, F: Frail.

The carbohydrate and plant protein intake tended to be lower in the frail male group than in the robust male group, but the difference did not reach statistical significance $(p=0.064,0.092$, respectively) (Figure 2A,C). Although the difference in total dietary fiber intake between the robust and frail groups was also marginal ( $p=0.059)$, the intake of soluble dietary fiber was significantly lower in the prefrail male group than in the robust male group (Figure 2F). Among various nutrients, the intake of potassium, iron, $\beta$-carotene (equivalent), vitamins B1, B2, B6, and C, folate, and pantothenic acid was lower in the prefrail male group than in the robust male group (Table 4). The intake of vitamin B2 and B6 was lower in the frail male group than in the robust group. In terms of food groups, the intake of confectioneries was significantly lower in the frail male group than in the robust male group.

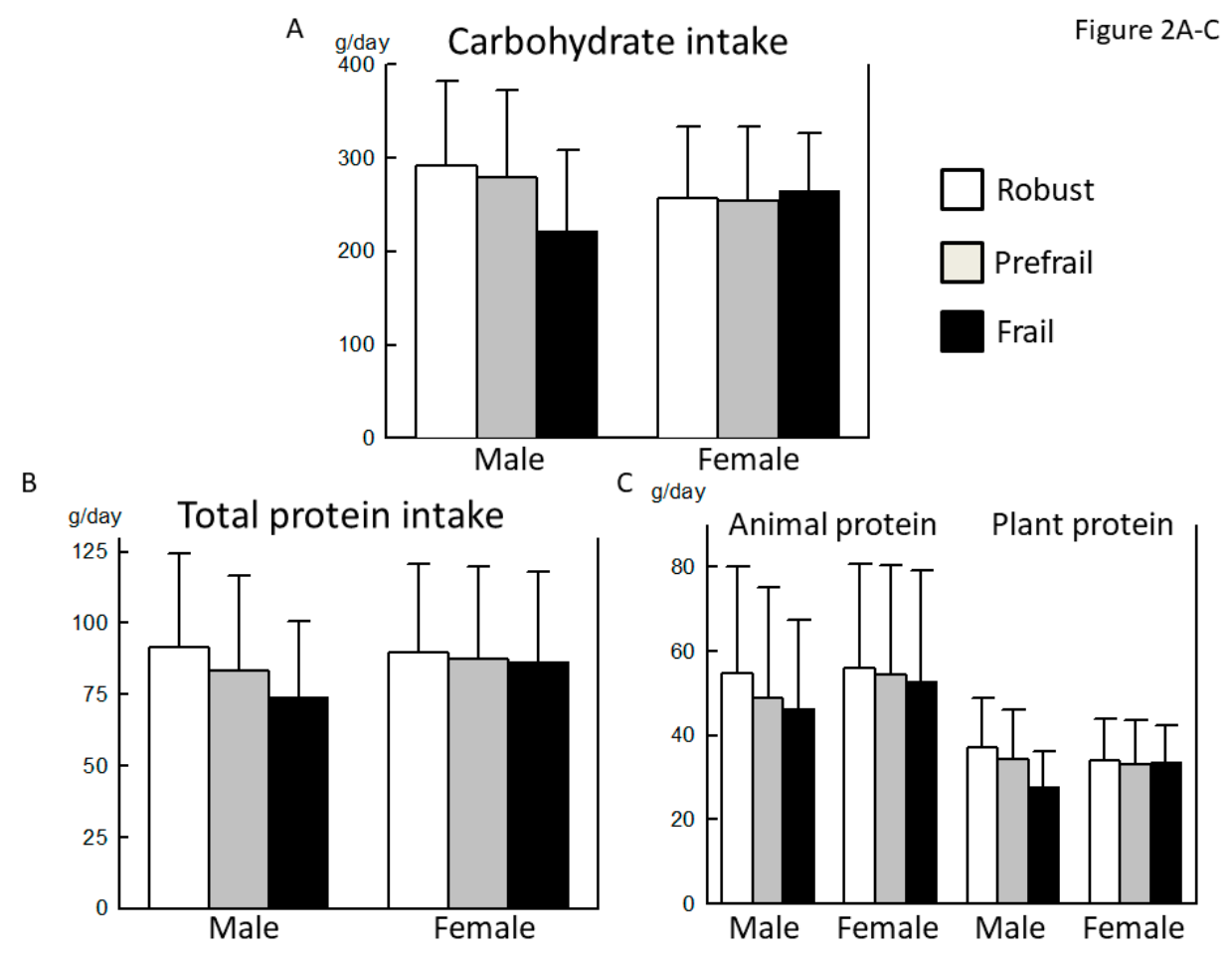

Figure 2. Cont. 
Figure 2D-F

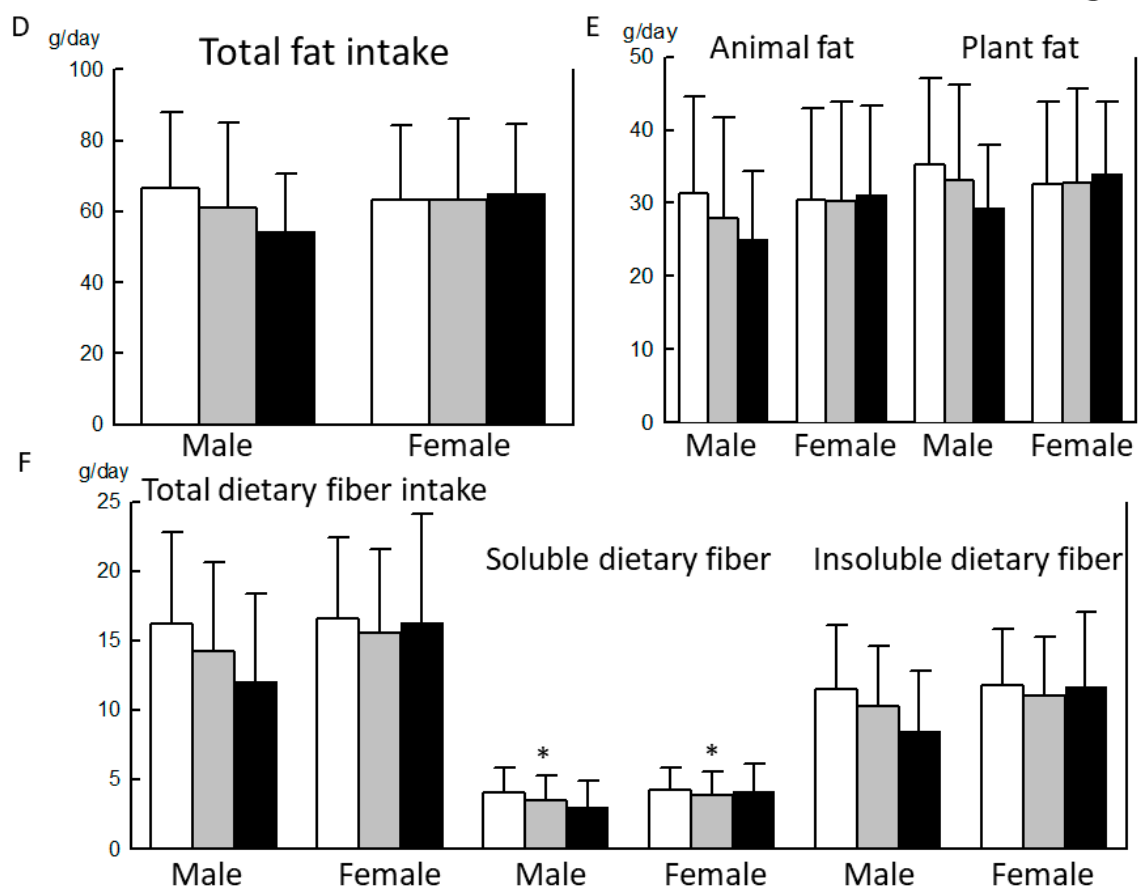

Figure 2. Comparison of macronutrient intake between robust, prefrail, and frail individuals, diagnosed by the Japanese version of the Cardiovascular Health Study. (A): Carbohydrate intake, (B): Total protein intake, (C): Animal and plant protein intake, (D): Total fat intake, (E): Animal and plant fat intake, and (F): Total, soluble, and insoluble dietary fiber intake. $*: p<0.05$ vs. Robust.

As shown in Table 5, age was the highest and the height was the lowest in the frail female group between the three groups. BMI was significantly higher in the prefrail female group, compared with that in the robust female group. In addition, the frail female group had the lowest exercise status. The proportion of chronic liver disease, CKD, and gastrointestinal disease was significantly higher and that of dyslipidemia was significantly lower in the frail female group.

Table 5. Basic characteristics and dietary habits among frail, prefrail, and robust women, as diagnosed by the J-CHS criteria.

\begin{tabular}{|c|c|c|c|c|}
\hline & Robust $(n=227)$ & Prefrail $(n=294)$ & Frail $(n=23)$ & $p$-Value \\
\hline Age (years) & $72.2 \pm 5.4$ & $72.3 \pm 5.5$ & $76.5 \pm 7.8$ & 0.002 (R vs. F) 0.002 (P vs. F) \\
\hline Height $(\mathrm{cm})$ & $152.2 \pm 5.2$ & $151.2 \pm 5.4$ & $147.6 \pm 7.6$ & $<0.001$ (R vs. F) 0.007 (P vs. F) \\
\hline $\mathrm{BW}(\mathrm{kg})$ & $51.0 \pm 6.4$ & $51.7 \pm 7.5$ & $49.1 \pm 8.9$ & n.s. \\
\hline BMI $\left(\mathrm{kg} / \mathrm{m}^{2}\right)$ & $22.0 \pm 2.5$ & $22.6 \pm 3.0$ & $22.5 \pm 4.2$ & 0.042 (R vs. P) \\
\hline $\mathrm{SBP}(\mathrm{mmHg})$ & $138.8 \pm 17.3$ & $140.0 \pm 17.4$ & $142.6 \pm 17.8$ & n.s. \\
\hline $\mathrm{DBP}(\mathrm{mmHg})$ & $80.5 \pm 10.7$ & $80.8 \pm 10.2$ & $80.4 \pm 9.2$ & n.s. \\
\hline MMSE score & $28.4 \pm 1.7$ & $28.4 \pm 1.8$ & $27.6 \pm 1.9$ & n.s. \\
\hline \multicolumn{5}{|l|}{ Smoking Habit } \\
\hline Never & $215(94.7)$ & $269(91.5)$ & $22(95.7)$ & \\
\hline Past & $7(3.1)$ & $19(6.5)$ & 0 & \\
\hline Current & $5(2.2)$ & $6(2.0)$ & $1(4.3)$ & n.s. \\
\hline Alcohol drinking & $65(28.6)$ & $89(30.3)$ & $2(8.7)$ & n.s. \\
\hline Exercise habit & $227(100.0)$ & $121(41.2)$ & $3(13.0)$ & $<0.001$ \\
\hline \multicolumn{5}{|l|}{ Comorbidities } \\
\hline Hypertension & $90(39.6)$ & $129(43.9)$ & $14(60.9)$ & n.s. \\
\hline $\mathrm{DM}$ & $20(8.8)$ & $24(8.2)$ & $4(17.4)$ & n.s. \\
\hline Dyslipidemia & $50(22.0)$ & $94(32.0)$ & $3(13.0)$ & 0.012 \\
\hline Chronic liver disease & $5(2.2)$ & $10(3.4)$ & $3(13.0)$ & 0.021 \\
\hline CKD & $1(0.4)$ & $12(4.1)$ & $2(8.7)$ & 0.009 \\
\hline CVD & $11(4.8)$ & $23(7.8)$ & $2(8.7)$ & n.s. \\
\hline Gastrointestinal disease & $8(3.5)$ & $18(6.1)$ & $4(17.4)$ & 0.017 \\
\hline Asthma/ COPD & $4(1.8)$ & $11(3.7)$ & $2(8.7)$ & n.s. \\
\hline
\end{tabular}


Table 5. Cont.

\begin{tabular}{|c|c|c|c|c|}
\hline & Robust $(n=227)$ & Prefrail $(n=294)$ & Frail $(n=23)$ & $p$-Value \\
\hline Thyroid disease & $8(3.5)$ & $17(5.8)$ & $2(8.7)$ & n.s. \\
\hline Osteoporosis & $29(12.8)$ & $60(20.4)$ & $4(17.4)$ & n.s. \\
\hline RA and collagen disease & $7(3.1)$ & $4(1.4)$ & $1(4.3)$ & n.s. \\
\hline \multirow[t]{2}{*}{ History of cancer } & $10(4.4)$ & $15(5.1)$ & $3(13.0)$ & n.s. \\
\hline & Robust $(n=227)$ & Prefrail $(n=294)$ & Frail $(n=23)$ & $p$-value \\
\hline Energy intake (kcal/day) & $1978 \pm 551$ & $1962 \pm 585$ & $2010 \pm 472$ & n.s. \\
\hline $\begin{array}{c}\text { Energy intake/BW (kcal/day/kg) } \\
\text { Macro- and micronutrients }\end{array}$ & \multicolumn{3}{|c|}{ Macro- and micronutrients } & n.s. \\
\hline Carbohydrate (\% energy/day) & $52.0 \pm 7.0$ & $51.8 \pm 7.5$ & $53.3 \pm 5.9$ & n.s. \\
\hline Total protein ( $\%$ energy/day) & $18.1 \pm 3.3$ & $17.7 \pm 3.4$ & $16.8 \pm 3.2$ & n.s. \\
\hline Fat (\% energy /day) & $28.6 \pm 4.8$ & $28.8 \pm 5.1$ & $29.0 \pm 4.5$ & n.s. \\
\hline Sodium (g/day) & $4.78 \pm 1.53$ & $4.73 \pm 1.61$ & $4.72 \pm 1.57$ & n.s. \\
\hline Potassium (g/day) & $3.57 \pm 1.16$ & $3.33 \pm 1.21$ & $3.47 \pm 1.42$ & 0.048 (R vs. P) \\
\hline Calcium (mg/day) & $826 \pm 302$ & $776 \pm 301$ & $819 \pm 381$ & n.s. \\
\hline Magnesium (mg/day) & $334 \pm 106$ & $317 \pm 110$ & $325 \pm 123$ & n.s. \\
\hline Phosphorus (mg/day) & $1414 \pm 492$ & $1357 \pm 504$ & $1379 \pm 529$ & n.s. \\
\hline Iron (mg/day) & $10.6 \pm 3.5$ & $10.1 \pm 3.6$ & $10.4 \pm 3.7$ & n.s. \\
\hline Zinc (mg/day) & $9,78 \pm 3.11$ & $9.61 \pm 3.16$ & $9.56 \pm 3.07$ & n.s. \\
\hline Manganese (mg/day) & $3.92 \pm 1.12$ & $3.83 \pm 1.18$ & $3.87 \pm 1.14$ & n.s. \\
\hline Copper (mg/day) & $1.38 \pm 0.42$ & $1.33 \pm 0.43$ & $1.41 \pm 0.45$ & n.s. \\
\hline Retinol equivalent ( $\mu \mathrm{g} /$ day) & $1080 \pm 732$ & $958 \pm 555$ & $1016 \pm 580$ & n.s. \\
\hline$\alpha$-Carotene $(\mu \mathrm{g} /$ day $)$ & $680 \pm 423$ & $633 \pm 445$ & $703 \pm 565$ & n.s. \\
\hline$\beta$-Carotene equivalent ( $\mu \mathrm{g} /$ day) & $6111 \pm 3083$ & $5657 \pm 3236$ & $6295 \pm 4494$ & n.s. \\
\hline Vitamin D ( $\mu \mathrm{g} /$ day) & $25.5 \pm 16.2$ & $23.9 \pm 15.8$ & $21.6 \pm 15.1$ & n.s. \\
\hline$\alpha$-Tocopherol (mg/day) & $9.80 \pm 3.33$ & $9.45 \pm 3.60$ & $10.52 \pm 4.70$ & n.s. \\
\hline$\gamma$-Tocopherol (mg/day) & $14.0 \pm 4.9$ & $14.0 \pm 5.4$ & $15.6 \pm 4.5$ & n.s. \\
\hline Vitamin $\mathrm{K}$ (mg/day) & $432 \pm 203$ & $401 \pm 205$ & $421 \pm 280$ & n.s. \\
\hline Vitamin B1 (mg/day) & $1.01 \pm 0.33$ & $0.97 \pm 0.35$ & $0.95 \pm 0.33$ & n.s. \\
\hline Vitamin B2 (mg/day) & $1.75 \pm 0.54$ & $1.67 \pm 0.57$ & $1.75 \pm 0.60$ & n.s. \\
\hline Niacin (mg/day) & $22.0 \pm 8.3$ & $21.2 \pm 8.7$ & $19.9 \pm 8.1$ & n.s. \\
\hline Vitamin B6 (mg/day) & $1.69 \pm 0.58$ & $1.61 \pm 0.61$ & $1.60 \pm 0.63$ & n.s. \\
\hline Vitamin B12 ( $\mu \mathrm{g} /$ day $)$ & $14.1 \pm 7.9$ & $13.8 \pm 8.4$ & $13.1 \pm 9.0$ & n.s. \\
\hline Folate ( $\mu \mathrm{g} /$ day $)$ & $499 \pm 181$ & $462 \pm 176$ & $480 \pm 224$ & 0.049 (R vs. P) \\
\hline Pantothenic acid (mg/day) & $8.31 \pm 2.65$ & $7.89 \pm 2.73$ & $8.08 \pm 2.62$ & n.s. \\
\hline Vitamin C (mg/day) & $193 \pm 78$ & $173 \pm 75$ & $180 \pm 103$ & 0.013 (R vs. P) \\
\hline Cryptoxanthin ( $\mu \mathrm{g} /$ day $)$ & $527 \pm 378$ & $474 \pm 380$ & $477 \pm 437$ & n.s. \\
\hline \multicolumn{5}{|l|}{ Food group (g/day) } \\
\hline Cereals & $340 \pm 137$ & $345 \pm 149$ & $376 \pm 134$ & n.s. \\
\hline Potatoes & $76.8 \pm 60.4$ & $65.7 \pm 55.5$ & $73.4 \pm 47.4$ & n.s. \\
\hline Sugar and sweeteners & $6.26 \pm 4.14$ & $5.94 \pm 4.78$ & $6.87 \pm 4.50$ & n.s. \\
\hline Soy products & $91.5 \pm 47.3$ & $87.0 \pm 49.6$ & $89.9 \pm 52.8$ & n.s. \\
\hline Total vegetables & $391 \pm 185$ & $372 \pm 185$ & $385 \pm 262$ & n.s. \\
\hline Fruits & $193 \pm 123$ & $170 \pm 116$ & $190 \pm 166$ & n.s. \\
\hline Fish and shellfish & $124 \pm 75$ & $120 \pm 80$ & $123 \pm 108$ & n.s. \\
\hline Meats & $79.5 \pm 50.4$ & $80.6 \pm 48.4$ & $58.7 \pm 23.2$ & n.s. \\
\hline Eggs & $44.3 \pm 26.0$ & $44.5 \pm 29.6$ & $60.1 \pm 42.4$ & 0.036 (R vs. F) 0.033 (P vs. F). \\
\hline Dairy products & $179 \pm 101$ & $169 \pm 104$ & $211 \pm 133$ & n.s. \\
\hline Oil & $10.4 \pm 5.2$ & $10.6 \pm 5.2$ & $11.7 \pm 4.8$ & n.s. \\
\hline Confectioneries & $70.1 \pm 53.8$ & $71.8 \pm 56.8$ & $59.2 \pm 39.5$ & n.s. \\
\hline
\end{tabular}

Mean \pm SD or number (\%), n.s.: not significant, R: Robust, P: Prefrail, F: Frail.

No statistically significant differences in carbohydrate, protein, and fat intake were observed between the three groups (Figure 2A-E). Although the difference in total dietary fiber and insoluble dietary fiber intake between the robust and prefrail female groups was marginal $(p=0.080,0.084$, respectively), the intake of soluble dietary fiber was significantly lower in the prefrail female group, compared with the robust female group (Figure 2F). Among various nutrients, the intake of potassium, vitamin C, and folate was lower in the prefrail female group than in the robust female group (Table 4). In terms of food groups, the intake of eggs was the highest in the frail female group. By contrast, the intake of meats tended to be lower in the frail female group $(p=0.098)$.

\subsection{Reevaluation of Significant Associations of the Intakes of Soluble Dietary Fiber, Potassium, Folate, and Vitamin C with a Frail Status by ANCOVA}

Since decreased intakes of soluble dietary fiber, potassium, folate, and vitamin $\mathrm{C}$ were commonly observed in frail men by the KCL criteria, in prefrail men by the J-CHS criteria, and in prefrail women by the J-CHS criteria, we conducted an ANCOVA to determine significant differences in 4 dietary 
nutrients between the robust, prefrail, and frail groups, after excluding the possible confounding effects of baseline differences. An analysis of men revealed significant associations of the intakes of soluble dietary fiber, potassium, folate, and vitamin $\mathrm{C}$ with a frail status diagnosed by the J-CHS ( $p=0.035$, 0.023. 0.012 , and 0.007 , respectively) and that diagnosed by the KCL ( $p=0.008,0.011 .0 .007$, and 0.006 , respectively). In contrast, we did not observe associations of a frail status with any dietary factors except vitamin $C$ in women ( $p=0.128,0.137 .0 .107$, and 0.027 , respectively). Thus, we concluded that the sufficient intakes of dietary fiber, potassium, folate, and vitamin $\mathrm{C}$ may be critical for preventing frailty in men.

\section{Discussion}

This study provides several novel and interesting findings, with regards to the dietary habits of older adults with frailty, which include (1) The patterns of the dietary habits differed between older adults with prefrailty or frailty and healthy individuals, depending on the diagnostic criteria of frailty that was used. (2) The problem in dietary habits appears from the stage of prefrailty diagnosed by the J-CHS. By contrast, it is not clear at the stage of prefrailty by the KCL. (3) The pattern of dietary habits in older adults with frailty or prefrailty varied significantly between men and women. The nutritional problem was more remarkable in frail or prefrail men. (4) Significant associations of the intakes of soluble dietary fiber, potassium, folate, and vitamin $C$ with a frail status were observed in men, and an association of the intake of vitamin $\mathrm{C}$ with a frail status in women, according to the J-CHS.

Frailty represents a complex of clinical syndrome, and is characterized by decreased physiological reserve and increased vulnerability to stressors, resulting from multiple impairments across different organs [1,2]. Frailty is caused by multiple factors, including aging-associated loss of skeletal muscle mass, reduced nutritional intake, and low physical activity [1,2]. In recent times, the neuropsychiatric status, including cognitive impairment and depression, as well as social conditions such as solitude, have been shown to contribute to frailty $[1,2,21]$. Therefore, numerous tools to measure the frailty status have been developed. These can be divided into two groups: the phenotype model and the accumulation of deficits model, as represented by the CHS criteria, and the Frailty index, respectively [21]. In this study, we used the J-CHS as the phenotype model, and the KCL as the accumulation of deficits model. The KCL was originally designed to screen Japanese community-dwelling elderly adults whose status could potentially progress to the point of requiring long-term care [16]. Satake et al. evaluated the adequacy of a diagnosis of frailty severity based on the KCL criteria or the CHS criteria and reported that the respective sensitivity and specificity of these methods were $70.3 \%$ and $78.3 \%$ for prefrail individuals and $89.5 \%$ and $80.7 \%$ for frail individuals [16]. In this study, the two frailty diagnoses had a concordance rate of approximately $60 \%$. As the KCL assesses the seven subdomains, including instrumental ADL, physical function, nutrition, oral function, socialization, memory, and mood [11,16], the impact of the physical aspect of frailty may become diluted by the domains of cognitive impairment, depression, and social frailty in individuals identified as frail using this model. We speculate that recent nutritional issues may more strongly affect the physical aspect of frailty than the neuropsychiatric and social aspects. Accordingly, the differences in basic characteristics and dietary habits between the robust and prefrail or frail groups, as identified using the two distinct sets of criteria, seemed acceptable. Our results suggest that nutritional interventions should be customized according to the characteristics of the diagnostic tool. Furthermore, uniform nutritional instructions, such as increases in the calorie and protein intakes, might be redundant for the treatment and prevention of frailty and sarcopenia in older adults. Although the KCL includes two queries about the nutritional status and three about oral function $[11,16]$, the total score is used to diagnose frailty. Therefore, the J-CHS might more sensitively detect abnormal dietary habits in an earlier stage of the frailty cycle. Nutritional items should be extracted from the KCL prior to reevaluation, given the risk that this comprehensive frailty rating system might underestimate issues with a patient's dietary habits. Accordingly, in a recent KCL-based study, the authors evaluated the associations between the frequency of protein-rich food intake and 
the frailty subdomains of KCL [11]. Further studies are needed to conclude which frailty assessment tool can better detect potential nutritional disturbances in community-dwelling older adults.

In our study, the nutritional issue had a more remarkable effect in frail men, compared with frail women. Notably, the male subjects included higher numbers of current smokers and alcohol drinkers, compared with the female subjects (Table 1). The excess intake of these luxury grocery items might affect the nutritional statuses of men, consistent with previous studies demonstrating that alcohol consumption can limit nutrient absorption and bioavailability in the blood [22]. This finding suggests a need for increased daily living guidance concerning these luxury grocery items, especially for men. By contrast, women exhibited a significantly higher prevalence of sarcopenia and osteoporosis (Table 1). In other words, a sex-related discrepancy exists between the possible involvement of malnutrition in the development of frailty and the higher prevalence of some downstream hallmarks of frailty.

The population in this study appeared to be healthy and well-nourished and had a relatively high intake of protein (Figures 1 and 2). In such a population, the effect of insufficient nutritional intake on the development of frailty may have been affected by other factors. Additionally, inflammation, oxidative stress, and neurohumoral factors may contribute to the development of frailty $[23,24]$. Our results led us to speculate that men were more vulnerable to nutritional issues, whereas comorbidities might have a relatively greater effect on the development of frailty in women. Given the nature of frailty as a complex clinical syndrome comprising low physical activity, exhaustion, body weight loss, and other factors, the nutritional issue identified in this study might be more responsible for these components of frailty, rather than a low skeletal muscle mass or low bone density. We note the metabolisms of both bone and muscle are closely associated with sex hormones [25]. Furthermore, the prevalence of osteoporosis and sarcopenia may be affected by comorbid orthopedic diseases (e.g., osteoarthritis of the knee and lumbar spondylosis), most of which develop independently of the nutritional status [26]. These factors may also be responsible for the sex-related discrepancy between the involvement of malnutrition in the development of frailty and the prevalence of some downstream hallmarks of frailty. Therefore, discussions of sex differences in the pathophysiology of frailty should distinguish the osteoarticular and muscular components of frailty from other components.

Although self-administered dietary assessment methods, including questionnaires, cannot assess energy intake properly $[8,19]$, this study showed no difference in energy intake between robust, prefrail, and frail individuals in the three populations, except for men diagnosed by the KCL (Tables 2-5). This finding suggests that lower energy intake is responsible for decreased intakes of macronutrients in frail men according to the KCL and the frailty might develop independently of total energy intake. Previous studies, focusing on protein intake in elderly adults demonstrated that 1.0 to $1.2 \mathrm{~g} / \mathrm{kg} /$ day protein intake is minimal, and 1.3 to $1.5 \mathrm{~g} / \mathrm{kg} /$ day protein intake is recommended for elderly adults to perform endurance or resistance training [25]. More recently, Japanese investigators demonstrated close association between the frequency of protein-rich food intake, such as seafood and dairy products, and the prevalence of frailty diagnosed by KCL [11]. Although malnutrition, including lower protein intake, was observed in frail men diagnosed by KCL, we could not find any close association between protein intake and the prevalence of frailty in other groups. In the present study, protein intake of the participants was higher, compared with the general nutrient intake recommendations $[13,25]$. This may be because our participants had high physical performance levels and a good nutritional status. However, the importance of adequate protein intake for the prevention of frailty cannot be discounted.

By contrast, lower intake of soluble dietary fiber, potassium, vitamin C, and folate was commonly observed in prefrail or frail older adults, compared with the healthy people among the two different diagnostic methods for the robust, prefrail, and frail men (Figures 1 and 2, and Tables 2 and 4). In elderly adults whose intake of total energy and protein is sufficient, the variety of food groups may become more important. Dietary fibers have gained renewed interest as they are degraded by enteric bacteria in the colon into short chain fatty acids, which play an important role in maintaining the health of the colon and the whole body $[27,28]$. Recent papers suggested that elevated dietary fiber intake is closely 
associated with the health of elderly adults as well as longevity [29]. Our results were consistent with a previous paper from the Osteoporotic Fractures in Men (MrOS) study in the United States of America, which demonstrated that a higher intake of dietary fiber, but not of protein, significantly decreased the risk of prefrailty or frailty in community-dwelling older men [30]. To prevent the progression of frailty in older adults with sufficient energy and protein intake, the significance of consuming more dietary fiber should be clarified in future studies. In the frailty cycle, chronic inflammation, increased oxidative stress, and/or imbalances in the neurohumoral factors may contribute to the development of frailty independent of the general nutritional status $[23,24]$. Therefore, previous investigations demonstrated the association between frailty and micronutrients, especially vitamins and carotenoids, that may have antioxidant or anti-inflammatory properties $[9,15]$. Among various minerals and vitamins, lower intake of vitamin C and folate was frequently found in observational studies, including our study $[9,15]$. These results strongly suggest that consuming fruits and green and yellow vegetables is desirable to prevent frailty, in addition to preventing atherosclerosis.

This study has some limitations. First, due to its cross-sectional design, it was impossible to describe causal relationships. Longitudinal and interventional studies are required to quantitatively evaluate food intakes and to clarify the causal relationships in the future. Second, the survey was conducted in individuals who were capable of visiting our hospital for health check-ups using transportation means. In addition, we performed the dietary survey using a self-administered questionnaire. Therefore, our participants were relatively healthy. In fact, the proportion of frail participants was lower in this study than in previous studies performed in Japan [16,18]. It is often observed that frailty in older adults is accompanied with mild cognitive impairment [2,21]. Exclusion of individuals who displayed impaired cognitive function (MMSE score less than 24) may contribute to the lower prevalence of frailty in this study. Fourth, due to the lower prevalence of frailty in this study, it is possible that the significance of specific macronutrients or food group intake in frail older adults might become obscure due to statistical limitations. Finally, we failed to ask the participants questions about their marital status and whether they live alone or not. Eating alone may contribute to the development of frailty, at least in part, through enhancing nutritional imbalances.

In conclusion, our results demonstrate the association between frailty and dietary habits in rural community-dwelling older adults. In addition, our study suggests that attention should be paid to the diagnostic criteria of frailty and differences between sexes when nutritional interventions for older adults with frailty are planned. To prevent the progression of frailty in older adults who have sufficient energy and protein intake, a comprehensive nutritional management plan, including optimal intake of dietary fiber, minerals, and vitamins, should be developed in a longitudinal study in the near future.

Author Contributions: Research design, data collection, analysis, and writing of the manuscript: K.T., H.K., K.N., and K.S.; Participation in data collection: S.T., Y.W., M.I., K.S., M.A., H.M., Y.H., H.K., and S.S. All authors approved the final version of the manuscript.

Funding: This research was funded in part by the JSPS KAKENHI (Grant Number 16KT0012) (2016-2018), a grant for the support of collaborative investigation between Hyogo College of Medicine and Hyogo University of Health Sciences (2017-2018) (to Dr. Shinmura), and the grant for Good Practice to Establish Centers for Fostering Medical Researchers of the Future by Ministry of Education, Culture, Sports, Science and Technology (2015-2017) (to Hyogo College of Medicine).

Acknowledgments: The FESTA study team includes Hiroo Yoshikawa, Akira Okayama, Yoshinori Ohta, Mayuka Fukai, Shinichi Hirayama, Yukio Nogaki, Saki Shimizu, Masahiro Murakami, Megumi Yasuda, Shigefumi Murakami, Yoshiko Hatanaka, Atsushi Kondo, Beni Kira, Miyuu Ueno, Koji Yasuhira, Yukio Nogaki, Akane Inoue. Nobuhide Horii, Ayumi Sakuramoto-Sadakane, Masako Shiramizu, Masumi Nakanishi, Kana Hasegawa, Saori Sano, and Hideyuki Sugita.

Conflicts of Interest: The authors declare no conflict of interest. 


\section{References}

1. Bergman, H.; Ferrucci, L.; Guralnik, J.; Hogan, D.B.; Hummel, S.; Karunananthan, S.; Wolfson, C. Frailty: An emerging research and clinical paradigm-issues and controversies. J. Gerontol. A Biol. Sci. Med. Sci. 2007, 62, 731-737. [CrossRef] [PubMed]

2. Morley, J.E.; Vellas, B.; van Kan, G.A.; Anker, S.D.; Bauer, J.M.; Bernabei, R.; Cesari, M.; Chumlea, W.C.; Doehner, W.; Evans, J.; et al. Frailty consensus: A call to action. J. Am. Med. Dir. Assoc. 2013, 14, 392-397. [CrossRef]

3. Xue, Q.L.; Bandeen-Roche, K.; Varadhan, R.; Zhou, J.; Fried, L.P. Initial manifestations of frailty criteria and the development of frailty phenotype in the Women's Health and Aging Study II. J. Gerontol. A Biol. Sci. Med. Sci. 2008, 63, 984-990. [CrossRef] [PubMed]

4. Bonnefoy, M.; Berrut, G.; Lesourd, B.; Ferry, M.; Gilbert, T.; Guerin, O.; Hanon, O.; Jeandel, C.; Paillaud, E.; Raynaud-Simon, A.; et al. Frailty and nutrition: Searching for evidence. J. Nutr. Health Aging 2015, 19, 250-257. [CrossRef] [PubMed]

5. Yannakoulia, M.; Ntanasi, E.; Anastasiou, C.A.; Scarmeas, N. Frailty and nutrition: From epidemiological and clinical evidence to potential mechanisms. Metabolism 2017, 68, 64-76. [CrossRef] [PubMed]

6. Bartali, B.; Frongillo, E.A.; Bandinelli, S.; Lauretani, F.; Semba, R.D.; Fried, L.P.; Ferrucci, L. Low nutrient intake is an essential component of frailty in older persons. J. Gerontol. A Biol. Sci. Med. Sci. 2006, 61, 589-593. [CrossRef] [PubMed]

7. Kim, H.K.; Suzuki, T.; Saito, K.; Yoshida, H.; Kobayashi, H.; Kato, H.; Katayama, M. Effects of exercise and amino acid supplementation on body composition and physical function in community-dwelling elderly Japanese sarcopenic women: A randomized controlled trial. J. Am. Geriatr. Soc. 2012, 60, 16-23. [CrossRef]

8. Kobayashi, S.; Murakami, K.; Sasaki, S.; Okubo, H.; Hirota, N.; Notsu, A.; Fukui, M.; Date, C. Comparison of relative validity of food group intakes estimated by comprehensive and brief-type self-administered diet history questionnaires against $16 \mathrm{~d}$ dietary records in Japanese adults. Public Health Nutr. 2011, 14, 1200-1211. [CrossRef]

9. Lorenzo-Lopez, L.; Maseda, A.; de Labra, C.; Regueiro-Folgueira, L.; Rodriguez-Villamil, J.L.; Millan-Calenti, J.C. Nutritional determinants of frailty in older adults: A systematic review. BMC Geriatr. 2017, 17, 108. [CrossRef]

10. Rahi, B.; Colombet, Z.; Gonzalez-Colaco Harmand, M.; Dartigues, J.F.; Boirie, Y.; Letenneur, L.; Feart, C. Higher Protein but Not Energy Intake Is Associated with a Lower Prevalence of Frailty Among Community-Dwelling Older Adults in the French Three-City Cohort. J. Am. Med. Dir. Assoc. 2016, 17, 672.e7-672.e11. [CrossRef]

11. Yamaguchi, M.; Yamada, Y.; Nanri, H.; Nozawa, Y.; Itoi, A.; Yoshimura, E.; Watanabe, Y.; Yoshida, T.; Yokoyama, K.; Goto, C.; et al. Association between the Frequency of Protein-Rich Food Intakes and Kihon-Checklist Frailty Indices in Older Japanese Adults: The Kyoto-Kameoka Study. Nutrients 2018, 10, 84. [CrossRef]

12. Chevalier, S.; Gougeon, R.; Nayar, K.; Morais, J.A. Frailty amplifies the effects of aging on protein metabolism: Role of protein intake. Am. J. Clin. Nutr. 2003, 78, 422-429. [CrossRef] [PubMed]

13. Morais, J.A.; Chevalier, S.; Gougeon, R. Protein turnover and requirements in the healthy and frail elderly. J. Nutr. Health Aging 2006, 10, 272-283. [PubMed]

14. Coelho-Junior, H.J.; Rodrigues, B.; Uchida, M.; Marzetti, E. Low Protein Intake Is Associated with Frailty in Older Adults: A Systematic Review and Meta-Analysis of Observational Studies. Nutrients 2018, $10,1334$. [CrossRef] [PubMed]

15. Balboa-Castillo, T.; Struijk, E.A.; Lopez-Garcia, E.; Banegas, J.R.; Rodriguez-Artalejo, F.; Guallar-Castillon, P. Low vitamin intake is associated with risk of frailty in older adults. Age Ageing 2018, 47, 872-879. [CrossRef] [PubMed]

16. Satake, S.; Senda, K.; Hong, Y.J.; Miura, H.; Endo, H.; Sakurai, T.; Kondo, I.; Toba, K. Validity of the Kihon Checklist for assessing frailty status. Geriatr. Gerontol. Int. 2016, 16, 709-715. [CrossRef] [PubMed]

17. Fried, L.P.; Tangen, C.M.; Walston, J.; Newman, A.B.; Hirsch, C.; Gottdiener, J.; Seeman, T.; Tracy, R.; Kop, W.J.; Burke, G.; et al. Frailty in older adults: Evidence for a phenotype. J. Gerontol. A Biol. Sci. Med. Sci. 2001, 56, M146-M156. [CrossRef] [PubMed] 
18. Shimada, H.; Makizako, H.; Doi, T.; Yoshida, D.; Tsutsumimoto, K.; Anan, Y.; Uemura, K.; Ito, T.; Lee, S.; Park, H.; et al. Combined prevalence of frailty and mild cognitive impairment in a population of elderly Japanese people. J. Am. Med. Dir. Assoc. 2013, 14, 518-524. [CrossRef]

19. Kobayashi, S.; Honda, S.; Murakami, K.; Sasaki, S.; Okubo, H.; Hirota, N.; Notsu, A.; Fukui, M.; Date, C. Both comprehensive and brief self-administered diet history questionnaires satisfactorily rank nutrient intakes in Japanese adults. J. Epidemiol. 2012, 22, 151-159. [CrossRef]

20. Ministry of Education, C., Sports, Science and Technology. The 5th revision of Standard Food Composition Table of Japanese Foods (in Japanese). Available online: http://www.mext.go.jp/b_menu/shingi/gijyutu/ gijyutu3/houkoku/1298713.htm (accessed on 1 February 2012).

21. Shinmura, K. Cardiac Senescence, Heart Failure, and Frailty: A Triangle in Elderly People. Keio. J. Med. 2016, 65, 25-32. [CrossRef]

22. Lieber, C.S. The influence of alcohol on nutritional status. Nutr. Rev. 1988, 46, 241-254. [CrossRef] [PubMed]

23. Fedarko, N.S. The biology of aging and frailty. Clin. Geriatr. Med. 2011, 27, 27-37. [CrossRef]

24. Fulop, T.; Larbi, A.; Witkowski, J.M.; McElhaney, J.; Loeb, M.; Mitnitski, A.; Pawelec, G. Aging, frailty and age-related diseases. Biogerontology 2010, 11, 547-563. [CrossRef] [PubMed]

25. Bauer, J.; Biolo, G.; Cederholm, T.; Cesari, M.; Cruz-Jentoft, A.J.; Morley, J.E.; Phillips, S.; Sieber, C.; Stehle, P.; Teta, D.; et al. Evidence-based recommendations for optimal dietary protein intake in older people: A position paper from the PROT-AGE Study Group. J. Am. Med. Dir. Assoc. 2013, 14, 542-559. [CrossRef]

26. Vina, E.R.; Kwoh, C.K. Epidemiology of osteoarthritis: Literature update. Curr. Opin. Rheumatol. 2018, 30, 160-167. [CrossRef] [PubMed]

27. Holscher, H.D. Dietary fiber and prebiotics and the gastrointestinal microbiota. Gut Microbes 2017, 8, $172-184$. [CrossRef] [PubMed]

28. Koh, A.; De Vadder, F.; Kovatcheva-Datchary, P.; Backhed, F. From Dietary Fiber to Host Physiology: Short-Chain Fatty Acids as Key Bacterial Metabolites. Cell 2016, 165, 1332-1345. [CrossRef]

29. Cai, D.; Zhao, S.; Li, D.; Chang, F.; Tian, X.; Huang, G.; Zhu, Z.; Liu, D.; Dou, X.; Li, S.; et al. Nutrient Intake Is Associated with Longevity Characterization by Metabolites and Element Profiles of Healthy Centenarians. Nutrients 2016, 8, 564. [CrossRef]

30. Shikany, J.M.; Barrett-Connor, E.; Ensrud, K.E.; Cawthon, P.M.; Lewis, C.E.; Dam, T.T.; Shannon, J.; Redden, D.T.; Osteoporotic Fractures in Men (MrOS) Research Group. Macronutrients, diet quality, and frailty in older men. J. Gerontol. A Biol. Sci. Med. Sci. 2014, 69, 695-701. [CrossRef] 